University of Wollongong

Research Online

Faculty of Engineering - Papers (Archive)

Faculty of Engineering and Information

Sciences

2000

\title{
A computational technique for simulating ionization energy deposition by energetic ions in complex targets
}

I. Cornelius

University of Wollongong, iwan@uow.edu.au

Anatoly B. Rosenfeld

University of Wollongong, anatoly@uow.edu.au

P. D. Bradley

University of Wollongong

R. L. Maughan

Wayne State University, USA

Follow this and additional works at: https://ro.uow.edu.au/engpapers

Part of the Engineering Commons

https://ro.uow.edu.au/engpapers/15

\section{Recommended Citation}

Cornelius, I.; Rosenfeld, Anatoly B.; Bradley, P. D.; and Maughan, R. L.: A computational technique for simulating ionization energy deposition by energetic ions in complex targets 2000 .

https://ro.uow.edu.au/engpapers/15

Research Online is the open access institutional repository for the University of Wollongong. For further information contact the UOW Library: research-pubs@uow.edu.au 


\title{
A Computational Technique for Simulating Ionization Energy Deposition by Energetic Ions in Complex Targets
}

\author{
I. Cornelius, Student Member, IEEE, A. B. Rosenfeld, P. D. Bradley, Senior Member, IEEE, and \\ R. L. Maughan, Student Member, IEEE
}

\begin{abstract}
An ion transport code was developed for simulating ionization energy deposition by energetic ions in sensitive volumes of complex structures. The code was used to simulate recent microdosimetry measurements performed with silicon-on-insulator (SOI) microdosimeters in Fast Neutron Therapy (FNT).
\end{abstract}

\section{INTRODUCTION}

$\mathbf{M}$ ICRODOSIMETRY is a topic of interest to both the space radiation effects, and the radiobiology communities. It aims to measure a characteristic of an ion field (exposing either electronic devices or biological cells) related to the observed effects (e.g., SEU or cell death). This characteristic is the spectrum of linear energy transfer values (LET, $d E / d x$ ). An approximation to this spectrum may be determined by considering charge deposition events in a microscopic volume subject to the ion field [1]. The LET of an ion traversing the microscopic volume is approximated by the quantity lineal energy. The lineal energy is defined as the quotient of the energy deposited in the volume and the mean chord length of the volume [1].

Traditionally, measurement of lineal energy spectra for the purposes of radiobiology in radiation oncology and space research has been made using tissue equivalent, proportional counters (TEPC) [1]. There are however several disadvantages in measuring lineal energy spectra with TEPC's [1]. A number of these have been overcome by using reversed biased, silicon-on-insulator diode arrays with microscopic sensitive volumes as microdosimeters [2]-[5]. Silicon microdosimetry studies using SOI devices in radiation oncology have been reported previously [2]-[5]. In these studies, silicon microdosimeters were used to measure lineal energy spectra in such radiation oncology modalities as boron-neutron capture therapy (BNCT) [3], [4] and Proton therapy [2]. Recently the technique has been applied to measurements in fast neutron therapy (FNT). Preliminary results of these FNT measurements are presented in this paper.

When developing new techniques such as silicon microdosimetry, it is desirable to have a theoretical prediction of

Manuscript received January 24, 2000. This work was supported in part by the Australian Department of Industry, Science and Technology.

I. Cornelius, A. B. Rosenfeld, and P. D. Bradley are with the Department of Engineering Physics, University of Wollongong, Wollongong, NSW, 2522 Australia (e-mail: \{ic02; Anatoly; Peter_Bradley\}@uow.edu.au).

R. L. Maughan was with the Department of Radiation Oncology, Wayne State University, Detroit, MI, 48201 USA. He is now with the Department of Radiation Oncology, University of Pennsylvania, Philadelphia, PA 19104 USA.

Publisher Item Identifier S 0018-9499(00)11167-0. detector response. To simulate measurements, we require an ion transport code that is able to consider a sufficiently general geometry to model complex device structure. For the purposes of microdosimetry in radiobiology we require a code that allows transport of the complex ion fields characteristic of space and radiation oncology environments. Several codes exist, for example PRISM [6], which allow a flexible target geometry definition but are not flexible in their ability to consider different ion fields. There are codes such as TRIM [7] that allow transport of complex ion fields. Yet these are not very flexible in their target geometry definition. An aim of this work was to attempt to bridge this gap by developing an ion transport technique to address our needs for a simulation tool in silicon microdosimetry. The code developed is called transport of ions in complex targets (TICOTS). A second aim was to use the code to simulate microdosimetry measurements in FNT using the SOI microdosimeter.

\section{MethodS}

\section{A. TICOTS Code Description}

The TICOTS code transports energetic ions in complex targets. The code provides a frequency distribution of ionization energy deposition events in the sensitive volume (SV) of the target. A general description of the code is provided below.

1) Ion Field and Target Definition: A separate Monte Carlo, "generator" function is interfaced with the TICOTS code. This function is used for ion field definition and provides flexibility for considering the ion fields of different radiation oncology modalities. The ions are defined in a manner common to many ion transport codes. A number of parameters are specified for each ion: atomic number, atomic mass, energy, position, and direction cosines.

The complex target is modeled as a system of right-angled, parallelepiped (RPP) voxels in Cartesian coordinates. The TICOTS program must have access to an input file defining each voxel of the structure in terms of the following parameters:

- A flag defining if ions originate from the voxel, (whether it is a "generation voxel").

- The coordinates of the lower corner of the RPP voxel, proximal to the origin.

- The coordinates of the upper corner of the RPP voxel, distal to the origin. (These two points define six planes enclosing the voxel). 
- Voxel material: type, mass density, elemental composition and stoichiometry.

- A numerical value specifying the sensitivity status of the voxel. Zero for a noncharge collecting voxel or a value between zero and one for the efficiency of a charge collecting voxel.

2) Ion Transport: Within the simulation loop, a call is made to the generator function. An ion is sampled in terms of the parameters described in Section II-A-1. The ion is subsequently transported through the structure.

As the ion is transported through a particular voxel of the structure, energy loss and scatter due to elastic interactions with nuclei are monitored. With the methodology of Biersack [7] an energy dependent flight path is calculated for the ion in the voxel material. The ion is then transported to this new position taking into account ionization energy losses. The ion energy and trajectory following elastic scatter are then updated. If the flight path is such that the scatter point lies outside the voxel, a boundary search routine is initiated. The ion is transported to the boundary and a scatter interaction at the boundary is considered.

If the generator function returns details of an ion of atomic number not previously encountered, stopping powers are calculated for each material in the structure using the method of Biersack [7]. From this, a range function is formulated. This gives the range energy relation of the ion neglecting elastic scatter interactions. With this range energy function the program calculates ionization energy loss over the free flight paths between elastic scatter events.

For each ion history a tally is made of ion energy loss contributing to energy deposition in voxels which are part of the sensitive volume. (In each voxel the ion energy loss is corrected for by the charge collection efficiency of the voxel). This tally holds the energy deposition "event" for the ion and is recorded. Each ion is transported until it either stops within the target, or crosses the volume enclosing all sensitive voxels.

The transport code continues to call the generator function and transport ions until the number of events registered by the sensitive volume reaches that specified for good statistics. A frequency distribution of events is then established and used to construct a lineal energy spectrum [1]. This may then be compared to experimental results.

Currently the code allows definition of only one sensitive volume, however a simple adjustment can be made to consider several independent sensitive volumes.

The calculation of charge deposition in a voxel assumes ionization energy loss to be equivalent to energy deposition. This is based on the assumption that the finite range of secondary electrons, ion energy loss straggling and nuclear reactions may be neglected. For the simulation in this paper, we are considering a proton field with a mean energy of around $10 \mathrm{MeV}$. This field is incident upon a SV with approximate dimensions $10 \mu \mathrm{m} \times$ $10 \mu \mathrm{m} \times 10 \mu \mathrm{m}$. The range of secondary electrons in silicon for $1-10 \mathrm{MeV}$ protons is approximately $0.1-1 \mu \mathrm{m}$ [8]. Hence this assumption is reasonably satisfied for the current case. This assumption does however, place a limitation on the voxel sizes, and ion energies considered in a simulation using TICOTS [8].

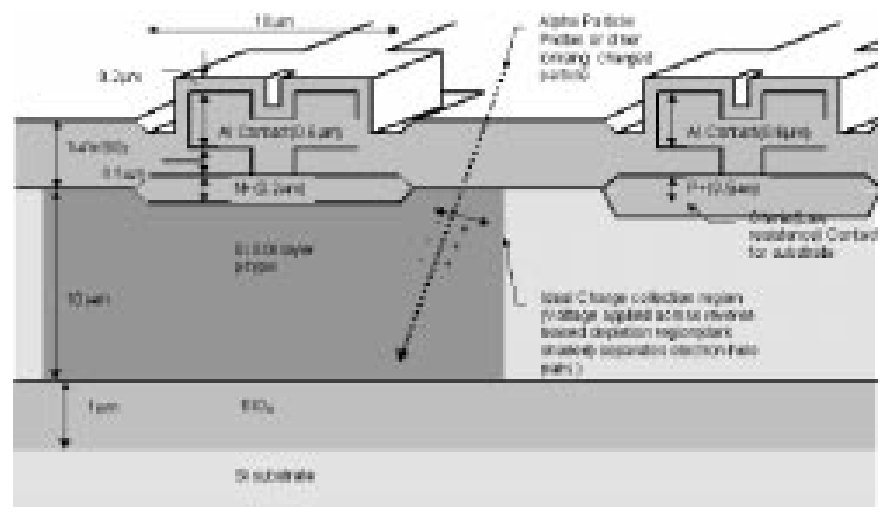

Fig. 1. Cross section of diode structure with approximate dimensions.

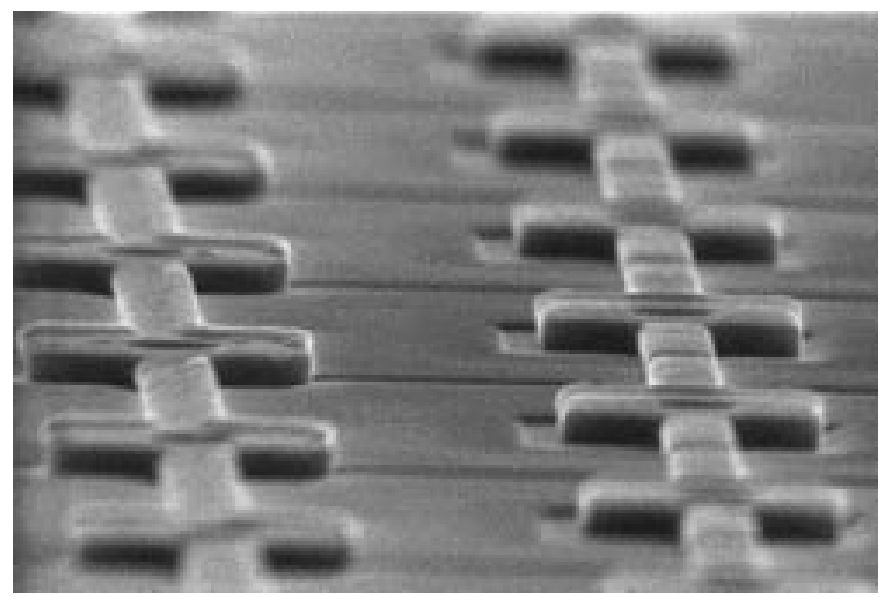

Fig. 2. SEM image of a portion of the $10 \mu \mathrm{m} \times 10 \mu \mathrm{m}$ diode array. (Note: Raised sections are aluminum tracks connecting contacts so that all diodes are electrically in parallel. The width of the image is about $23 \mu \mathrm{m}$.)

\section{B. SOI Microdosimeter Description}

As previously reported [2], [3], [5], the SOI microdosimeter consists of an array of reversed biased, $p-n$ junctions as illustrated in Figs. 1 and 2. Each diode has a junction size of $10 \mu \mathrm{m}$ $\times 10 \mu \mathrm{m}$ with each array consisting of $120 \times 40=4800$ diodes. The total size of each diode array was $30 \mathrm{~mm} \times 30 \mathrm{~mm}$, an array area of approximately $0.044 \mathrm{~cm}^{2}$. The SOI structures were fabricated on a bonded SOI wafer with $10 \mu \mathrm{m}$ thickness.

As previously reported [2], a radiation hard hybrid AMPTEK A-250 preamplifier was connected to the SOI chip in close proximity. The electronic readout circuit was optimized to reduce electronic noise and hence enable the measurement of low LET events in the microdosimeter. A noise measurement of this system was performed using Am-241, $60 \mathrm{keV}$ X-rays. This demonstrated a full width at half maximum of approximately $4.7 \mathrm{keV}$ under room temperature, in good agreement with theoretical simulations. In this mode the A-250 preamplifier was in the same radiation field as the microdosimeter. Preliminary testing of the A-250 was performed on a $1 \mathrm{MeV}$ neutron beam. Experiments were performed on the ( $\mathrm{p}, \mathrm{Li})$ neutron source at the Australian Nuclear Science and Technology Organization, Lucas Heights, Australia. Circuit performance was satisfactory up to a neutron fluence of approximately $10^{12} \mathrm{~cm}^{-2}$. 


\section{Measurement of a Lineal Energy Spectrum in FNT with SOI Microdosimeters}

Experimental measurements were made at the Gershenson Radiation Oncology Center, Harper Hospital using the super conducting cyclotron FNT facility [10]. Neutrons are produced via $48.5 \mathrm{MeV}$ deuteron bombardment of a Beryllium target. Beam definition is achieved using tungsten multi-rod collimators.

The microdosimeter was located at a depth of $2.5 \mathrm{~cm}$ in a water phantom with a source to surface distance (SSD) of $1830 \mathrm{~mm}$, on the axis of the $10 \mathrm{~cm} \times 10 \mathrm{~cm}$ beam. The microdosimeter was situated in a lucite envelope with wall thickness $5 \mathrm{~mm}$ to avoid water penetration. The microdosimeter was oriented with the array surface parallel with the phantom surface (normal to incident neutrons). The phantom was irradiated and the frequency distribution of charge deposition events in the SOI device monitored in the form of an MCA output.

The purpose of the experiment was to measure the lineal energy spectrum of the secondary charged particle field in phantom in FNT. A comparison could then be made with measurements performed with a traditional TEPC to further demonstrate the viability of the silicon microdosimetry technique in radiation oncology.

\section{Simulation of SOI Microdosimeter in FNT}

We wished to use the TICOTS code to simulate the SOI microdosimeter measurements in a water phantom subject to a FNT beam. As stated, the TICOTS code requires a generator function to sample an ion field. To a first approximation the secondary charged particle field of fast-neutrons in water may be modeled by the recoil proton component [1], [9]. A Monte Carlo code was developed to model the production of recoil protons by neutrons of an arbitrary energy spectrum irradiating a water phantom. (Monte Carlo Definition Of a Recoil Proton Field, MCDORPF). The neutron field is assumed to be of uniform flux, normally incident on a single hydrogenous voxel.

The MCDORPF code samples the neutron field and transports the neutrons through the hydrogenous voxel. Elastic scatter interactions are then monitored. For each interaction a recoil proton results. Its details (as outlined in Section II-A-1) are returned to the TICOTS code for transport through the target structure. Via this interface, the response of a SOI microdosimeter to a recoil proton field may be predicted.

The MCDORPF code requires the energy spectrum of the neutron beam as input. The FNT beam at this facility was previously characterized [10]. The spectrum at isocenter (the phantom surface) was used as input to the code and is shown in Fig. 3. The cross section data for elastic scatter of neutrons with hydrogen used by the code MCDORPF were obtained from the Korean Atomic Energy Research Institute (KAERI) [11].

The charge collection efficiency as a function of position in the SOI device was determined using ion microprobe and alpha beam spectroscopy as previously reported [2], [5]. It was found that there was a small variation in charge collection efficiency of the diodes from regions nearest the diode junction (0.86) to regions furthest (0.79) the junction. Using the average charge collection efficiency of 0.82 was considered adequate. The SOI

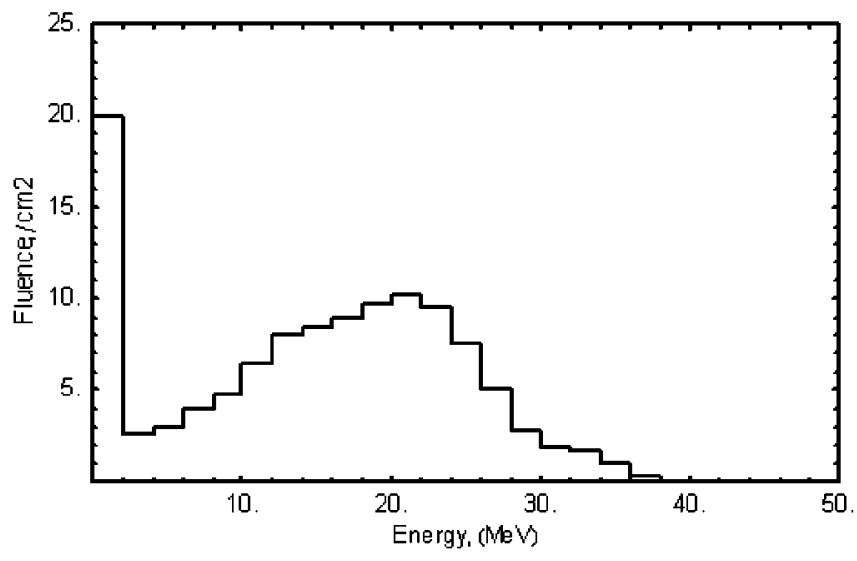

Fig. 3. Neutron energy spectrum at the isocenter of the Harper FNT facility [10].

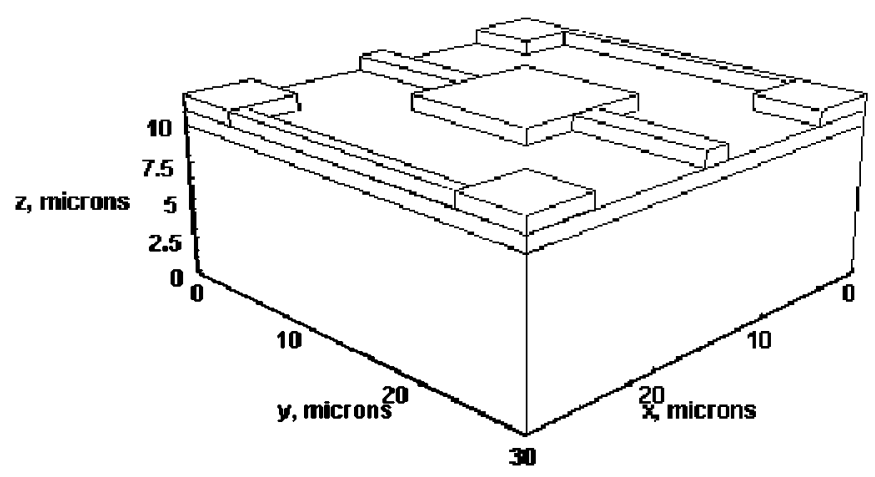

Fig. 4. Voxel definition of SOI microdosimeter used in simulation of FNT measurements. Note the overlying hydrogenous voxel (water phantom) is omitted from the figure.

sensitive volume was thus modeled as a single silicon voxel of $10 \mu \mathrm{m}$ thickness. Fig. 4 shows the simple RPP voxel model of a single diode used in the TICOTS simulation. A $10 \mu \mathrm{m}$ thick silicon voxel with a $1 \mu \mathrm{m}$ silicon oxide over-layer is considered. Above the silicon oxide are raised, $1 \mu \mathrm{m}$ thick voxels of aluminum. These represent the contacts and tracks connecting the diodes in parallel. The $2.5 \mathrm{~cm}$ of water phantom and polyethylene envelope were modeled as a single voxel of suitable hydrogen atomic density.

\section{E. Presentation of Lineal Energy Spectra}

The initial results of the simulation and experiment are frequency distributions of energy deposition events, $f(E)$. The mean chord length of the sensitive volume, as previously reported, is approximately $8.2 \mu \mathrm{m}$ [2]. Consequently, the frequency distribution of lineal energy events, $f(y)$ may be calculated. A dose probability density distribution, $d(y) \alpha y f(y)$ is then formed [1], [9]. This distribution emphasizes the fact that higher lineal energy events deposit a greater dose to the SV. In this form, equal areas under the curve represent equal doses to the SV. Lineal energy events may span several orders of magnitude. Hence it is customary to display the distribution with a logarithmic abscissa.

$$
\int d(y) d y \equiv \log (10) \int y d(y) d(\log y)
$$




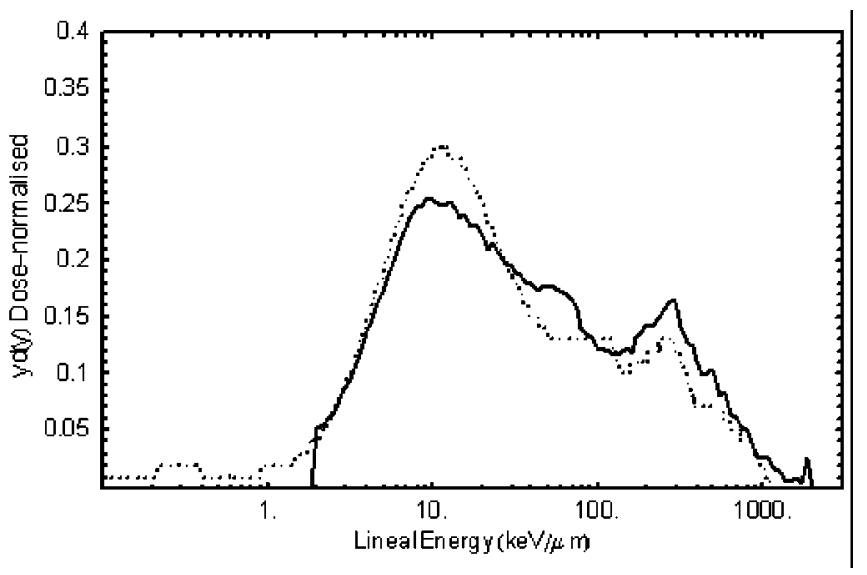

Fig. 5. Lineal energy dose distribution measured using the $10 \mu \mathrm{m}$ SOI silicon microdosimeter (solid line) and the TEPC (dotted line). Both measurements are at depth $2.5 \mathrm{~cm}$ in phantom, along the central axis of the beam. The plots are dose normalized, representing the fraction of dose to the microdosimeter sensitive volume per logarithmic interval of lineal energy.

Equation (1) exhibits the rationale for the final distribution being plotted as $y d(y)$ versus $\log (y)$ in the need to maintain the proportionality of area under the curve, and dose to the SV.

\section{RESULTS}

Prior to discussing results obtained in this study it will be beneficial to review a detailed analysis of a microdosimetric spectrum obtained in a previous study by Kota [12]. A measurement was performed using a spherical tissue equivalent proportional counter TEPC [12]. The TEPC aims to simulate a unit density tissue volume of $2 \mu \mathrm{m}$ diameter. The measurement was performed at the Harper hospital FNT facility under identical experimental and beam conditions. The dose normalized lineal energy spectrum of the SOI measurement, and that of the TEPC are illustrated in Fig. 5. The reader should note we are attempting to make a direct comparison between the LET spectrum measured in a tissue equivalent gas, to that measured in silicon. Using a rationale similar to that reported for the case of BNCT [4], the mean chord length of the silicon volume was scaled to facilitate agreement in the lineal energy of the proton peak.

The prominent feature of the microdosimetric spectrum is the recoil proton peak situated at about $10-15 \mathrm{keV} / \mu \mathrm{m}$. The position of the proton peak is dependent upon the mean neutron energy [9], the peak shifts to higher lineal energies with a decrease in mean neutron energy. The peak is also characterized by a "proton edge" at approximately $130 \mathrm{keV} / \mu \mathrm{m}$ corresponding to the maximum LET of protons in the SV material. For lineal energies greater than that of the proton edge the spectrum is composed of events from high LET ions. These are ascribed to alpha particles and heavy ion recoils produced via reactions of neutrons with the atomic constituents of the phantom [1], [9], [12], [13] and to a lesser extent the microdosimeter sensitive volume. At lineal energies below $10 \mathrm{keV} / \mu \mathrm{m}$, there is a contribution to the spectrum from photoelectrons. The neutron beam is contaminated by gamma radiation [12] produced by neutron interaction with the tungsten collimating rods. Photoelectron production in the sensitive volume may result in lineal energy events up to

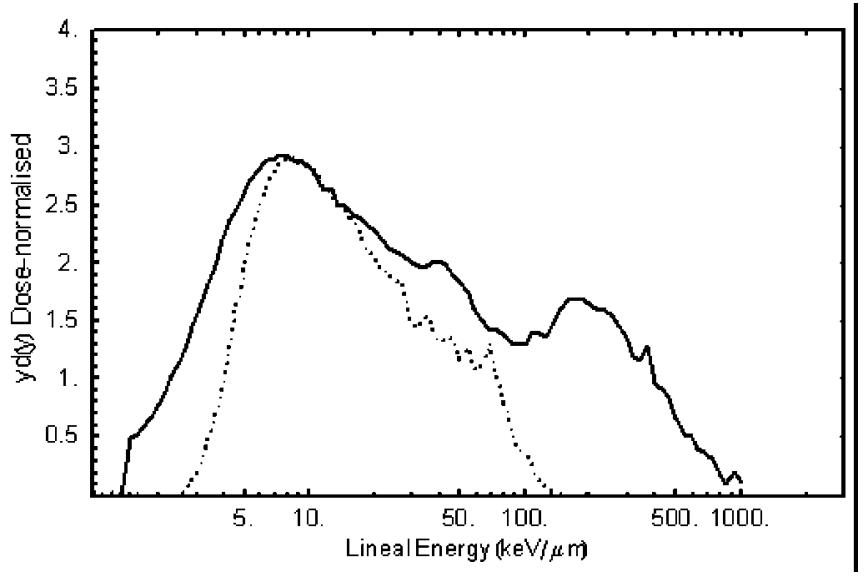

Fig. 6. Lineal energy spectra for simulated (dotted line) and experimental (solid line) results.

approximately $10 \mathrm{keV} / \mu \mathrm{m}[1]$. The relative contribution above $2 \mathrm{keV} / \mu \mathrm{m}$ is expected to be small in comparison to the proton component.

Differences between the TEPC spectrum and that of the SOI microdosimeter may be explained as follows. The geometry of the sensitive volumes of the microdosimeters is quite different. The SOI microdosimeter has a $10 \mu$ m thick RPP voxel whereas the TEPC simulates a $2 \mu$ m diameter sphere. Consequently there is an increased variance, and maximum chord length in the SOI microdosimeter. This leads to an increased spread in the observed spectrum and an increase in the likelihood of apparently large LET events as seen in Fig. 5. Furthermore a larger number of ions (particularly heavy ions) do not completely traverse the SOI sensitive volume because of its larger size [13]. This leads to the heavy ion recoil contribution extending to lower lineal energies, indicated by the "shoulder" on the proton peak at around $50 \mathrm{keV} / \mu \mathrm{m}$ in Fig. 5.

The results of the simulation and measurement are illustrated in Fig. 6. Both plots were normalized over the lineal energy range of $5-20 \mathrm{keV} / \mu \mathrm{m}$. In this region, lineal energy events could be assumed to originate from recoil protons alone. Events from other components of the ion field (photoelectrons, heavy ions) are considered to be negligible [1], [9], [12] at these lineal energies.

In the range of lineal energies, $5-50 \mathrm{keV} / \mu \mathrm{m}$ the spectrum is dominated by proton events [12]. There is agreement between experimental and simulated results in this region. A minor discrepancy related to the low lineal energy component of the peak exists. As mentioned, a component of the experimental spectrum is expected to result from photoelectrons. The generator function models recoil proton production alone and does not model the production of photoelectrons. This results in an under-estimate of the lineal energy spectrum in this lineal energy range.

Additionally, the generator function does not model the production of alpha particles and heavy ion recoils. Hence the simulation results do not display the peak in the lineal energy range $100-1000 \mathrm{keV} / \mu \mathrm{m}$ present in the experimental spectrum. There is also a discrepancy between experimental and theoretical results on the recoil proton peak in the region $20-100 \mathrm{keV} / \mu \mathrm{m}$. 
This is ascribed to heavy ions (not modeled in the current simulation) stopping in the sensitive volume and appearing as lower lineal energy events.

\section{CONCLUSIONS}

A silicon microdosimetric measurement was performed in a phantom subject to irradiation by a FNT beam. The results compared well with those achieved using a conventional TEPC. This demonstrates the validity of silicon based microdosimetry in FNT radiation oncology. Future development of a secondgeneration SOI microdosimeter is planned. A reduced mean chord length variance and a more defined, smaller sensitive volume are expected to facilitate a closer agreement with TEPC results. Application of the SOI microdosimeter to radiobiological studies relevant to space research is also to be pursued.

A computational technique was developed for simulating energy deposition in complex structures by energetic ions. The TICOTS code was developed to predict the SOI microdosimeter response to the ion fields of radiation oncology. Preliminary comparison of experimental and simulated results for the case of FNT was promising. Future code developments will be directed toward improving the model of the secondary ion field in FNT. This is to be achieved by incorporating the production of heavy ion recoils and photoelectrons into the generator function. The importance of neutron and proton interactions with the silicon sensitive volume will be investigated. A treatment of secondary electron tracking will be implemented to remove the limitation on voxel size as described in Section II-A-2. Use of a more general geometry such as combinatorial geometry in the TICOTS code will be addressed.

\section{REFERENCES}

[1] A. J. Waker, "Principles of experimental microdosimetry," Radiation Protection Dosimetry, vol. 61, pp. 297-300, 1995.

[2] A. B. Rosenfeld, P. D. Bradley, I. Cornelius, G. I. Kaplan, B. J. Allen, J. B. Flanz, M. Goitein, A. V. Meerbeeck, J. Schubert, J. Bailey, Y. Takada, A. Maruhashi, and Y. Hayakawa, "A new silicon detector for microdosimetry applications in proton therapy," IEEE Trans. Nucl. Sci., no. 4 2000.

[3] P. D. Bradley, A. B. Rosenfeld, B. J. Allen, J. Corderre, and J. Capela, "Performance of silicon microdosimetry detectors in boron neutron capture therapy," Radiation Research, vol. 151, pp. 235-243, 1999.

[4] P. D. Bradley and A. B. Rosenfeld, "Tissue equivalence correction for silicon microdosimetry detectors in BNCT," Med. Phys., vol. 25, pp. 2220-2225, 1998 .

[5] P. D. Bradley, A. B. Rosenfeld, M. Bailey, M. Reinhard, K. K. Kee, A. Saint, D. Jamieson, F. Lemeilleur, and S. Satoh, "Charge collection and radiation hardness of a SOI microdosimeter for space and medical applications," IEEE Trans. Nucl. Sci., vol. 45, pp. 2700-2710, 1998.

[6] M. K. Oldfield and C. I. Underwood, "PRISM-A tool for modeling proton energy deposition in semi-conductor materials," IEEE Trans. Nucl. Sci., vol. 43, pp. 2715-2723, 1996.

[7] J. P. Biersack and L. G. Haggmark, "A Monte Carlo computer program for the transport of energetic ions in amorphous targets," Nuclear Instruments and Methods, vol. 174, pp. 257-269, 1980.

[8] F. A. Cucinotta, R. Katz, J. W. Wilson, and R. R. Dubey, "Heavy ion track-structure calculations for radial dose in arbitrary materials,", NASA Tech. Memo. 3497, 1995.

[9] The International Commission on Radiation Units and Measurements, "Microdosimetry,", ICRU Report, vol. 36, 1983.

[10] T. D. Bohm, P. M. DeLuca Jr., R. L. Maughan, D. T. L. Jones, and A. Lennox, "Monte Carlo calculations to characterize the source for neutron therapy facilities," Med. Phys., vol. 26, pp. 783-792, 1999.

[11] Korean Atomic Energy Reserach Institute, "ENDFPLOT (computer program),", http://hpngp01.kaeri.re.kr/CoN/endfplot.shtml, 1999.

[12] C. Kota and R. L. Maughan, "Microdosimetric specification of the radiation quality of a d(48.5) + be fast neutron therapy beam produced by a superconducting cyclotron," Med. Phys., vol. 23, pp. 1591-1599, 1996.

[13] R. S. Caswell and J. J. Coyne, "Microdosimetric spectra and parameters of fast neutrons," in Fifth Symp. Microdosimetry, Verbania, Palanza, Italy, 1975. 